\title{
One-Step, Wash-free, Nanoparticle Clustering-Based Magnetic Particle Spectroscopy Bioassay Method for Detection of SARS-CoV-2 Spike and Nucleocapsid Proteins in the Liquid Phase
}

\author{
Kai Wu, ${ }^{*}{ }^{\perp}$ Vinit Kumar Chugh, ${ }^{\perp}$ Venkatramana D. Krishna, ${ }^{\perp}$ Arturo di Girolamo,
} Yongqiang Andrew Wang, Renata Saha, Shuang Liang, Maxim C-J Cheeran,* and Jian-Ping Wang*

Cite This: ACS Appl. Mater. Interfaces 2021, 13, 44136-44146

Read Online

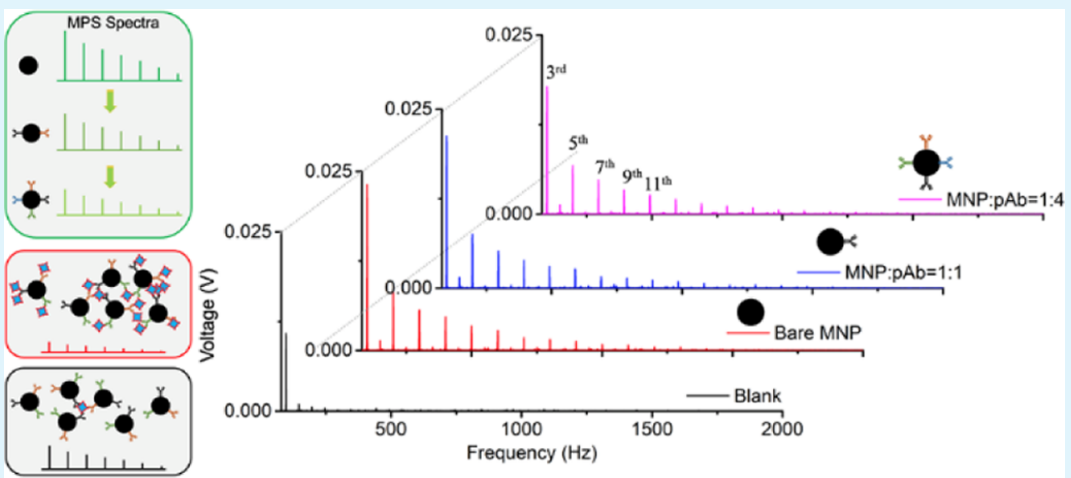

ABSTRACT: With the ongoing global pandemic of coronavirus disease 2019 (COVID-19), there is an increasing quest for more accessible, easy-to-use, rapid, inexpensive, and high-accuracy diagnostic tools. Traditional disease diagnostic methods such as qRTPCR (quantitative reverse transcription-PCR) and ELISA (enzyme-linked immunosorbent assay) require multiple steps, trained technicians, and long turnaround time that may worsen the disease surveillance and pandemic control. In sight of this situation, a rapid, one-step, easy-to-use, and high-accuracy diagnostic platform will be valuable for future epidemic control, especially for regions with scarce medical resources. Herein, we report a magnetic particle spectroscopy (MPS) platform for the detection of severe acute respiratory syndrome coronavirus 2 (SARS-CoV-2) biomarkers: spike and nucleocapsid proteins. This technique monitors the dynamic magnetic responses of magnetic nanoparticles (MNPs) and uses their higher harmonics as a measure of the nanoparticles' binding states. By anchoring polyclonal antibodies (pAbs) onto MNP surfaces, these nanoparticles function as nanoprobes to specifically bind to target analytes (SARS-CoV-2 spike and nucleocapsid proteins in this work) and form nanoparticle clusters. This binding event causes detectable changes in higher harmonics and allows for quantitative and qualitative detection of target analytes in the liquid phase. We have achieved detection limits of $1.56 \mathrm{nM}$ (equivalent to $125 \mathrm{fmole}$ ) and $12.5 \mathrm{nM}$ (equivalent to 1 pmole) for detecting SARS-CoV-2 spike and nucleocapsid proteins, respectively. This MPS platform combined with the one-step, wash-free, nanoparticle clustering-based assay method is intrinsically versatile and allows for the detection of a variety of other disease biomarkers by simply changing the surface functional groups on MNPs.

KEYWORDS: COVID-19, SARS-CoV-2, spike protein, nucleocapsid protein, magnetic nanoparticle, magnetic particle spectroscopy, disease diagnostics

\section{INTRODUCTION}

Severe acute respiratory syndrome coronavirus 2 (SARS-CoV2 ), a novel human corona virus, is the causative agent of ongoing coronavirus disease 2019 (COVID-19) pandemic. It was first identified in patients with acute respiratory illness in Wuhan, China, in December 2019 and soon after became a public health emergency of international concern. ${ }^{1}$ Since its emergence, SARS-CoV-2 infected 167 million people worldwide and caused 3.48 million deaths (as of May 25, 2021). The clinical presentation of SARS-CoV-2 infection ranges from asymptomatic or mild flu-like symptoms to fatal pneumonia and acute respiratory illness. ${ }^{2}$ The SARS-CoV-2 belongs to the genus betacoronavirus of the family Coronaviridae. They are enveloped, single-stranded, positive-sense RNA viruses with a

Received: August 2, 2021

Published: September 9, 2021 


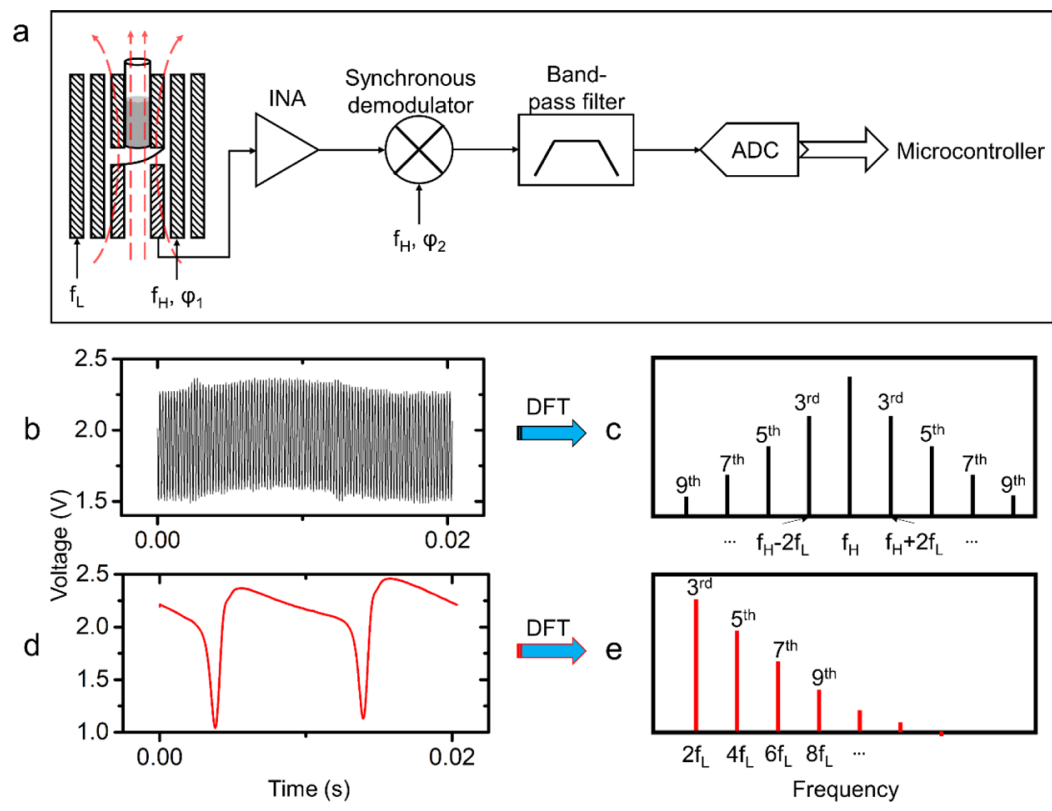

Figure 1. (a) Schematic diagram of the MPS system with a one-stage lock-in implementation. (b) Time-domain voltage signal collected from pickup coils after the INA amplification stage and (d) voltage signal post lock-in implementation. (c,e) MPS spectra from (b,d) after discrete Fourier transform (DFT), respectively.

genome size of approximately $29.9 \mathrm{~kb}^{3-5}$ The SARS-CoV-2 is about $60-140 \mathrm{~nm}$ in diameter and consists of 4 structural proteins and 16 nonstructural proteins. ${ }^{6}$ The structural proteins such as spike $(S)$, membrane $(M)$, and envelope (E) are associated with the viral envelope, and the nucleocapsid (N) protein forms the capsid outside genomic RNA.

Early diagnosis is critical for clinical management of patients and controlling the spread of COVID-19 by isolating infected patients. Several nucleic acid detection tests targeting N, E, S, or RNA-dependent RNA polymerase genes of the virus are commercially available for the diagnosis of COVID-19. ${ }^{7-9}$ Viral RNA detection by quantitative reverse transcription polymerase chain reaction (RT-qPCR) is currently the gold standard test for SARS-CoV-2 infection. ${ }^{10,11}$ Although this nucleic acid amplification test is highly sensitive with the limit of detection as low as 1 RNA copy $/ \mu \mathrm{L}$, RT-PCR needs expensive laboratory instrument, skilled technicians, and days to get the result. Therefore, there is an urgent need for a simple, rapid, sensitive, and accurate point-of-care diagnostic platform that can detect SARS-CoV-2 directly from clinical samples. The antigen detection test can directly detect SARSCoV-2 in respiratory specimens by detecting virus specific antigens. ${ }^{12-14}$ However, some of the commercially available antigen detection tests have very low sensitivity. ${ }^{15}$

Magnetic particle spectroscopy (MPS) is one of the most promising candidates for rapid, inexpensive, and high-accuracy bioassays. $^{16-22}$ It is a homogeneous biosensing tool that monitors the dynamic magnetic responses of magnetic nanoparticles (MNPs) in the liquid phase..$^{23-26}$ By applying AC magnetic fields to MNPs, the magnetic moments of MNPs follow the time-varying external field directions through a combined Néel and Brownian relaxation mechanism. ${ }^{16,18,27-29}$ The dominant relaxation of these two processes is dependent on the magnetic core sizes of MNPs (assuming unconstrained MNPs in liquid without surface binding of any chemical substances)..$^{30-32}$ The Brownian relaxation process is domi- nant for single-core iron oxide MNPs with core sizes above 20 $\mathrm{nm}$ (this critical size may vary for different magnetic materials of different magnetic properties). ${ }^{33-37}$ This relaxation process reflects the degree of freedom of physical rotational motion of MNPs. Conjugation of any chemical substances including protein molecules, aptamers, and other nonmagnetic materials onto MNPs can hinder or even block this Brownian relaxation. $^{23,27,38-45}$ As a result, this binding event causes a phase lag between magnetic moments and external AC fields (except at very low frequencies), and thus, weaker dynamic magnetic responses are observed from clustered MNPs. By exploiting this unique property of Brownian relaxationdominant MNPs, researchers have applied it along with the MPS platform for the detection of H1N1 virus, ${ }^{39}$ thrombin, ${ }^{38}$ staphylococcal toxins, ${ }^{20}$ SARS-CoV-2, ${ }^{23,46}$ botulinum neurotoxins $\mathrm{A}, \mathrm{B}$, and $\mathrm{E},{ }^{21}$ and so forth. Compared to the traditional optical, mechanical, and electrochemical sensing techniques, this magnetic sensing technique is immune to the background noise from biological samples that may interfere with the signal reading because most biological substrates are nonmagnetic (or paramagnetic) and MNPs are the only sources of the magnetic signal.

Our group has successfully built up both benchtop and portable MPS systems for one-step, wash-free bioassays. ${ }^{22,39}$ In this paper, we have modified the MPS system to include a onestage lock-in scheme for the advantages of down sampling and phase-sensitive rejection of noise. Herein, we report a one-step, wash-free, nanoparticle clustering-based MPS bioassay method for the detection of SARS-CoV-2 spike and nucleocapsid proteins in the liquid phase using this one-stage lock-in MPS system. By surface-conjugating polyclonal antibodies (pAbs) to MNPs, each MNP will be able to specifically bind to target protein molecules. On the other hand, each protein molecule has multiple epitopes that allow for the specific bindings of pAbs. Thus, the pAbs anchored to MNP surfaces and the nature of different epitopes from each protein molecule allow us to amplify the binding events caused by dynamic magnetic 


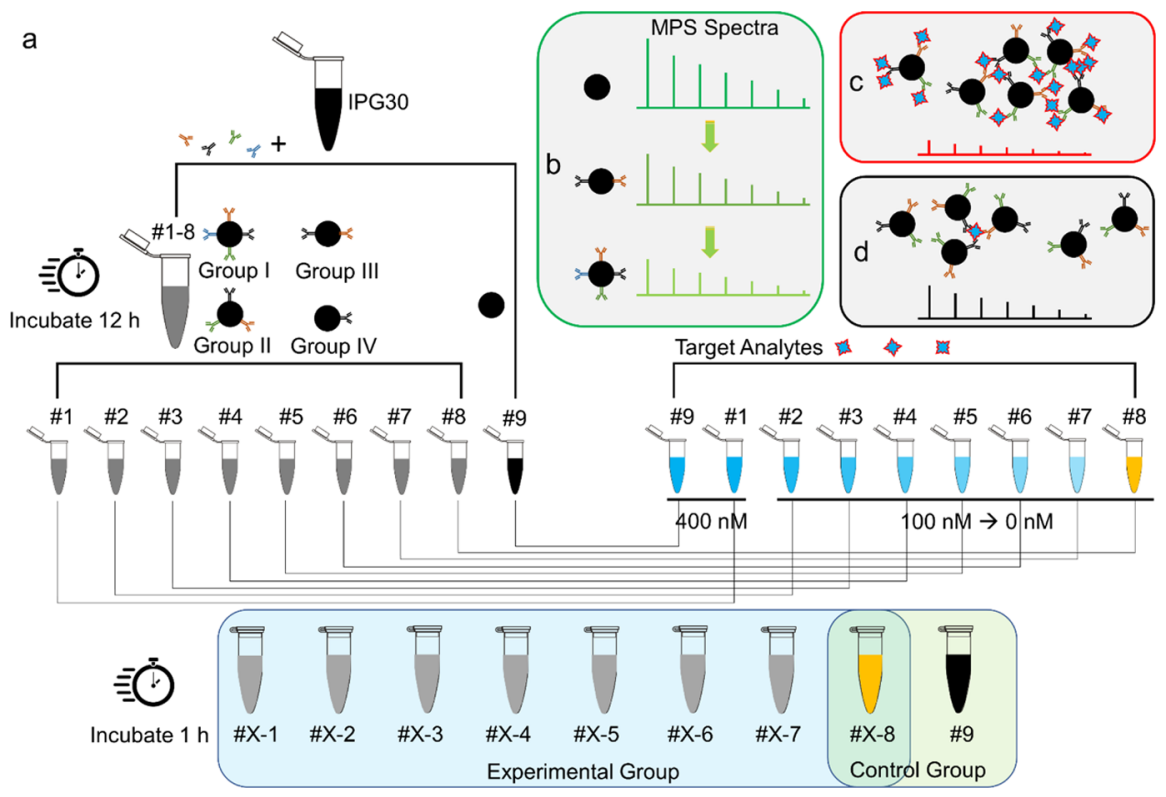

Figure 2. (a) Schematic view of samples prepared for one-step, wash-free, nanoparticle clustering-based MPS bioassays. For spike and nucleocapsid protein tests, four groups of samples are prepared by varying the amount of pAbs functionalized on MNPs. Group I: MNP/pAb = 1:4, Group II: $\mathrm{MNP} / \mathrm{pAb}=1: 3$, Group III: MNP/pAb = 1:2, and Group IV: MNP/PAb = 1:1. Vial \#9 is a negative control sample with bare MNPs (no pAbs functionalized). Spike and nucleocapsid proteins are prepared at varying concentrations from 400 to $0 \mathrm{nM}$. (b) Comparison of the MPS spectral intensities of MNPs functionalized with different numbers of pAbs. (c,d) Comparison of the MPS spectral intensities of surface functionalized MNPs in the presence of (c) high and (d) low concentrations/amounts of the target analytes.

response change. We demonstrate that this nanoparticle clustering-based detection method can detect target analytes by monitoring the dynamic magnetic responses of MNPs.

\section{MATERIALS AND METHODS}

2.1. Materials. The SARS-CoV-2 recombinant nucleocapsid protein (His tag, consists of 430 amino acids and predicts a molecular mass of $47.08 \mathrm{kDa}$, Cat: 40588 -V08B), the recombinant spike protein (RBD, His tag, consists of 234 amino acids with a molecular mass of $26.54 \mathrm{kDa}$, Cat: $40592-\mathrm{V} 08 \mathrm{H}$ ), the nucleocapsid antibody (polyclonal rabbit IgG, Cat: 40588-T62), and the spike RBD antibody (polyclonal rabbit IgG, Cat: 40592-T62) were purchased from Sino Biological Inc. The IPG30 MNPs were $30 \mathrm{~nm}$ iron oxide nanoparticles functionalized with protein $\mathrm{G}$, with a weight concentration of 1.7 $\mathrm{mg} / \mathrm{mL}$ and particle concentration of $57.8 \mathrm{nM}$, provided by Ocean NanoTech. The phosphate-buffered saline (PBS, Cat: 79378) was purchased from Sigma-Aldrich Inc.

2.2. Magnetic Property Characterization. $10 \mu \mathrm{L}$ of IPG30 MNP suspensions were pipetted onto a filter paper and air-dried. Then, the static magnetic hysteresis loops were collected on a Physical Properties Measurement System (PPMS, Quantum Design Inc.) to obtain the magnetic properties of these nanoparticles such as saturation $\left(M_{\mathrm{s}}\right)$ and coercivity $\left(H_{\mathrm{c}}\right)$. The static magnetic hysteresis loops of IPG30 MNPs were measured under external fields of $-5000-5000$ Oe and $-500-500 \mathrm{Oe}$, and the magnetic properties were analyzed in the Supporting Information S1.

2.3. One-Stage Lock-In MPS System. The MPS platform used in this work utilizes the frequency mixing approach where the excitation magnetic field consists of a low-frequency $\left(f_{\mathrm{L}}\right)$ component and a high-frequency $\left(f_{\mathrm{H}}\right)$ component. ${ }^{17,19-22,26,39,43} \mathrm{~A}$ low frequency field having $50 \mathrm{~Hz}$ frequency and 250 Oe amplitude and a highfrequency field having $5000 \mathrm{~Hz}$ frequency and 25 Oe amplitude were used for the magnetic excitation of the MNPs. In this work, the MPS system is modified with the addition of a one-stage lock-in implementation to improve the detection sensitivity of the system. Figure la shows the schematic representation of the sensing scheme used for decoding the MNP response signal in the one-stage lock-in MPS system. The signal from pick-up coils is amplified using a high- precision instrumentation amplifier, INA828 from Texas Instruments. Figure $1 \mathrm{~b}, \mathrm{c}$ shows the time-domain and frequency-domain representations of the MNP signal at this stage. The amplified signal is then processed by a one-stage lock-in implementation consisting of a synchronous demodulator followed by bandpass filtering. AD630 from Analog Devices is used for the synchronous demodulator application. Figure 1d,e depicts the corresponding time-domain and frequency-domain presentations of the MNP signal after the lock-in implementation stage. This one-stage lock-in MPS system helps remove the feedthrough signals corresponding to the excitation magnetic field frequencies and only records the dynamic magnetic responses of MNPs, hence improving the sensitivity. The filtered signal is sampled at a $100 \mathrm{KSPS}$ sampling rate and communicated to a laptop for postprocessing and data analysis. Each MPS reading for a vial contains 170,000 samples, which are collected over a period of roughly $2 \mathrm{~s}$. If multiple readings are required from a sample, we use a cooldown time of 3 min between the readings to ensure neither the coils nor the analogue driving circuitry heats up too much.

2.4. MPS-Based Bioassay Method. The MPS-based bioassays for the detection of target analytes in the liquid phase allows for onestep, wash-free assays that can be handled by a layperson without much technical training requirements. This method relies on the intentional interference on the Brownian relaxation of MNPs in liquid. When subjected to external AC magnetic fields (excitation fields), the magnetic moments of MNPs try to realign to external field directions through the Brownian relaxation. This process will be hindered if MNPs are surface-bound with polymers, protein molecules (such as antibody and antigen), aptamers, and so forth, ${ }^{18,23,26,38}$ fixed on a surface/substrate, ${ }^{20,21,46,47}$ or form nanoparticle clusters. $^{26,38,39}$ Figure $2 \mathrm{~b}$ shows a schematic view that by surface coating an increased number of antibodies, the dynamic magnetic responses of MNPs (in the form of MPS spectra, i.e., the amplitudes of higher harmonics) become weaker. In the presence of target analytes, the antibody-functionalized MNPs bind to these analytes and form nanoparticle clusters. As a result, this nanoparticle clustering events further weaken the dynamic magnetic response, thus lowering the harmonic amplitudes, as shown in Figure $2 \mathrm{c}$. On the other hand, if less target analytes are present, then less nanoparticle 
Table 1. Experimental and Control Group Designs for MPS-Based SARS-CoV-2 Spike (S) and Nucleocapsid (N) Protein Tests

\begin{tabular}{|c|c|c|c|c|}
\hline sample index & IPG30 MNP amount/vial & $\mathrm{S} / \mathrm{N} \mathrm{PAb}$ amount/vial & $\mathrm{MNP} / \mathrm{pAb}$ & $\mathrm{S} / \mathrm{N}$ protein amount/vial ${ }^{a}$ \\
\hline group I & 226 fmole & 904 fmole & $1: 4$ & $\# \mathrm{X}-1: 400 \mathrm{nM}(32 \text { pmole })^{b}$ \\
\hline vial $1-8$ & & & & \#X-2: $100 \mathrm{nM}$ (8 pmole) \\
\hline group II & 226 fmole & 678 fmole & $1: 3$ & \#X-3: $25 \mathrm{nM}$ (2 pmole) \\
\hline vial \#1-8 & & & & \#X-4: $12.5 \mathrm{nM}$ (1 pmole) \\
\hline group III & 226 fmole & 452 fmole & $1: 2$ & \#X-5: $6.25 \mathrm{nM}$ (500 fmole) \\
\hline vial \#1-8 & & & & \#X-6: $3.13 \mathrm{nM}$ (250 fmole) \\
\hline group IV & 226 fmole & 226 fmole & $1: 1$ & \#X-7: $1.56 \mathrm{nM}$ (125 fmole) \\
\hline vial \#1-8 & & & & $\# \mathrm{X}-8: 0 \mathrm{nM}(0$ fmole $)$ \\
\hline vial \#9 & 226 fmole & 0 fmole & NA & \#9: 400 nM (32 pmole) \\
\hline
\end{tabular}

${ }^{a} \mathrm{~S}$ and $\mathrm{N}$ stand for SARS-CoV-2 spike (S) and nucleocapsid (N) proteins, respectively. ${ }^{b} \mathrm{X}=\mathrm{I}$, II, III, and IV. There are four groups of samples prepared for $\mathrm{S}$ and $\mathrm{N}$ protein tests.

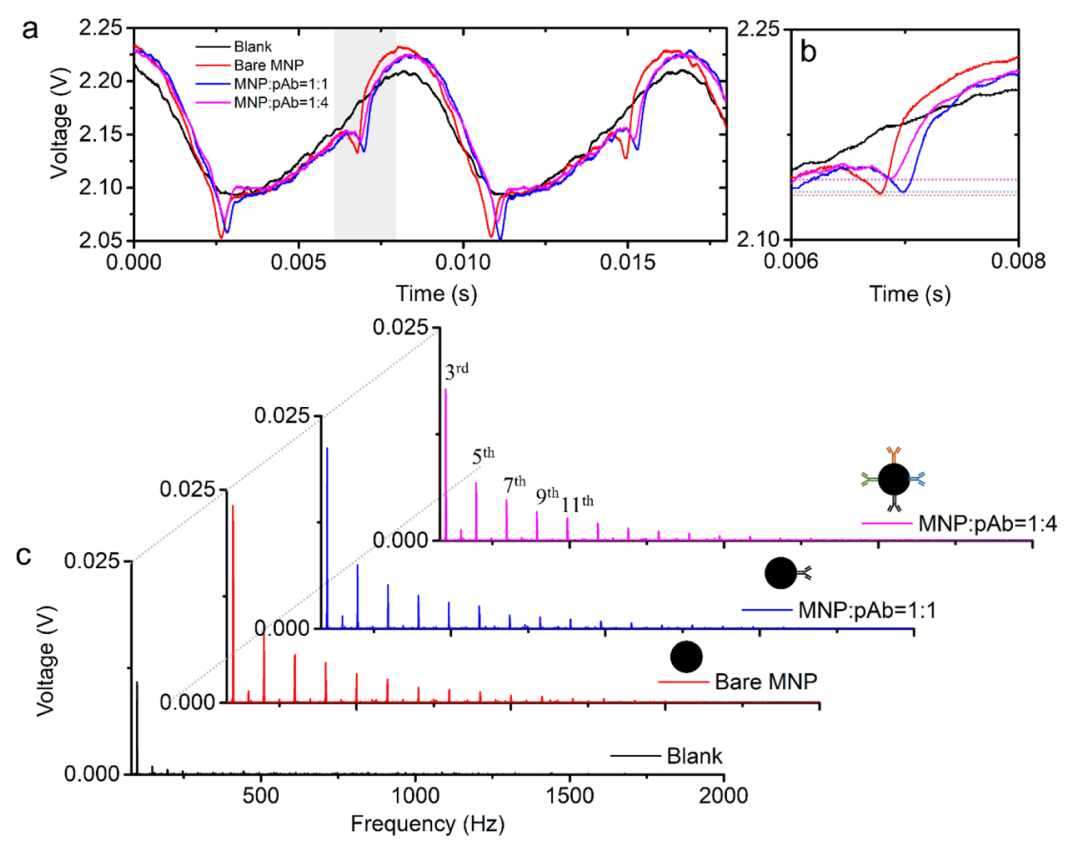

Figure 3. (a) Comparison of the time-domain voltage signal from the blank sample (floor signal), bare MNP, MNPs each coated with one pAb, and MNPs each coated with four pAbs. (b) Zoomed-in view of how the presence of MNPs and the surface functionalization of pAbs affect the realtime voltage signal. (c) Corresponding MPS spectra from (a). Higher harmonics such as the 3rd, 5th, 7th, 9th, and 11th are labeled in (c). Dotted lines have been added to help guide the view to compare the relative intensities of harmonics between the four sets of data.

clusters are formed and, as a result, the dynamic magnetic responses of MNPs yield stronger harmonics (Figure 2d).

2.5. Experimental and Control Groups. We first evaluated the detection limit of our one-stage lock-in MPS system for the detection of IPG30 MNPs. Samples containing a varying concentration/amount of IPG30 MNPs are prepared by two-fold dilutions, as shown in Table S1 from Supporting Information S2. Three independent MPS readings are taken from each sample, and the amplitudes of higher harmonics are summarized in Figure S2 from Supporting Information S2. Results show that the developed one-stage lock-in topology-based MPS system can detect as low as $266 \mathrm{ng}$ (equal to 9 fmole) of IPG30 MNPs, which equals to 512 -fold dilutions of the original concentration. Compared with our previous work, this modified MPS system has higher detection sensitivity. ${ }^{22,26,39}$ Thus, for the bioassays in this work, we used diluted IPG30 MNPs with the final particle concentration of $3.06 \mathrm{nM}$ and weight concentration of $90 \mu \mathrm{g} /$ $\mathrm{mL}$. This feasibility attempt allows us to explore inexpensive bioassays on our MPS platform for future high-volume tests at the users' ends and in regions with scarce medical resources. Furthermore, it is reported that for MPS-based homogeneous bioassays, lower MNP concentrations could improve the detection sensitivities of biomolecules. ${ }^{24,48}$

The design of one-step, wash-free, nanoparticle clustering-based MPS bioassays is highly relied on the surface functionalization of pAbs on MNPs. It has been reported in our previous work that the pAbs functionalized on MNPs can trigger the cross-linking between MNPs and target analytes, thus forming MNP clusters. ${ }^{39}$ In this work, the SARS-CoV-2 spike and nucleocapsid proteins have multiple epitopes that can be recognized by their corresponding pAbs. As a result, each protein molecule (i.e., spike and nucleocapsid) can bind to multiple pAbs from multiple MNPs. On the other hand, each MNP functionalized with multiple pAbs can bind to multiple protein molecules. A schematic example is given in Figure 2c.

As shown in Figure 2a, four experiment groups are designed by varying the number of pAby functionalized on MNPs: (1) Group I, $\mathrm{MNP} / \mathrm{pAb}=1: 4$ (i.e., in average, there are four $\mathrm{pAbs}$ functionalized on each MNP), (2) Group II, MNP/pAb = 1:3, (3) Group III, MNP/ $\mathrm{pAb}=1: 2$, and (4) Group IV, MNP/pAb = 1:1. Each group consists of eight surface-functionalized IPG30 MNP samples added with varying concentrations/amounts of spike or nucleocapsid proteins, as listed in Table 1. For the SARS-CoV-2 spike protein tests, we prepared four groups of samples along with one negative control sample (vial \#9) that contains bare IPG30 MNP without pAb coating (labeled as "bare MNP" in this work). In each group, the vials \#X-8 ( $\mathrm{X}=\mathrm{I}$, II, III, and IV) serve as the negative control that contain MNPs functionalized with a varying amount of pAbs, while $0 \mathrm{nM}$ protein (we replaced the protein with PBS buffer) is added. Thus, a 

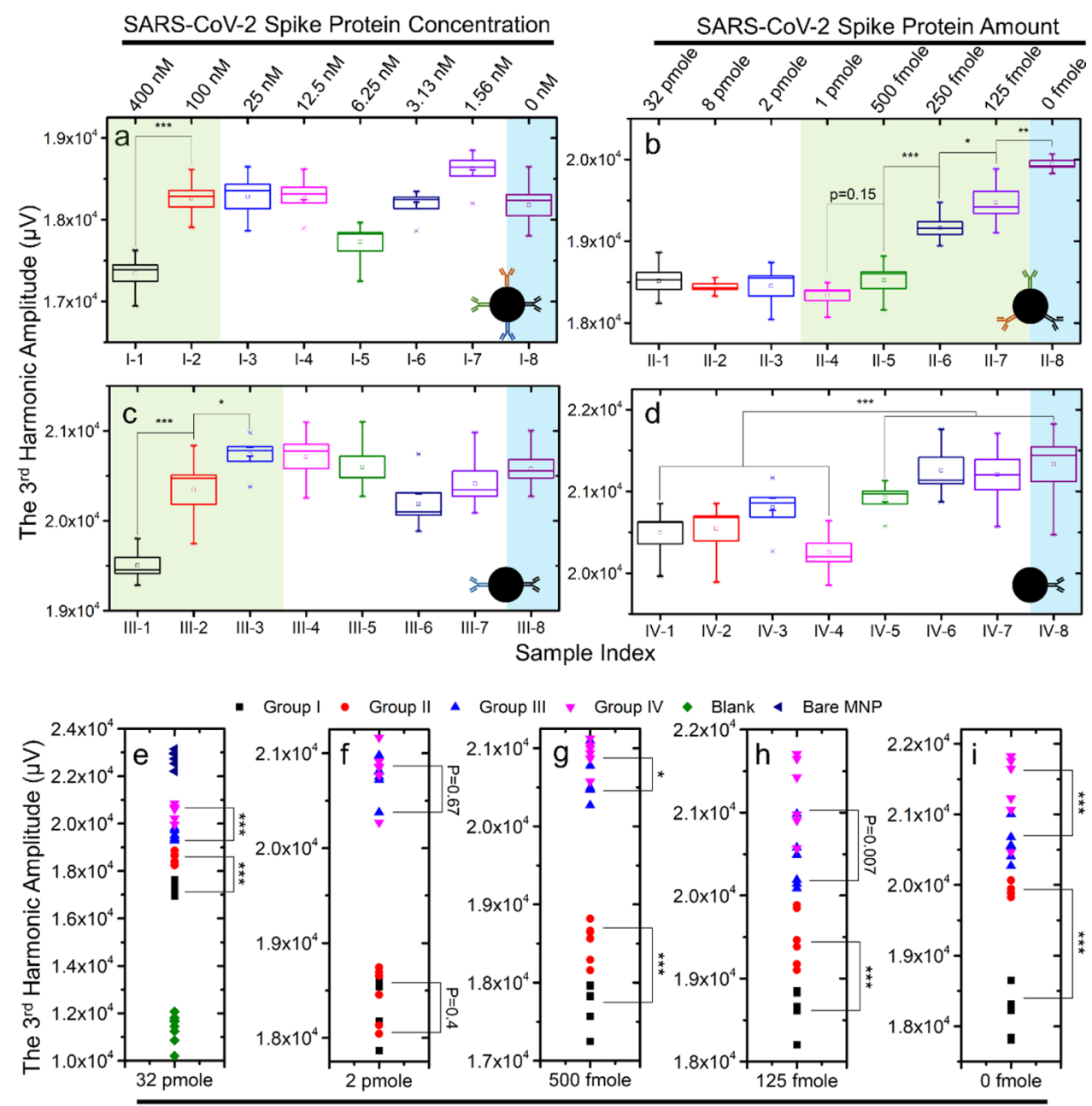

SARS-CoV-2 Spike Protein Amount

Figure 4. Concentration-response profiles of SARS-CoV-2 spike protein. (a-d) Box plots of the 3rd harmonic amplitudes from vials \#X-1 to \#X-8, where $\mathrm{X}=\mathrm{I}$, II, III, and IV, respectively. The highlighted green areas are the monotonic concentration-response regions, and the highlighted blue areas are the vials \#X-8, namely, control samples without the addition of target analytes. The middle horizontal line represents the averaged harmonic amplitude from six independent MPS readings, the top and bottom horizontal lines represent standard errors, and the asterisks represent highest and lowest harmonic amplitudes recorded among six independent readings. (e-i) Comparison of the 3rd harmonic amplitudes from vials \#X-1, \#X-3, \#X-5, \#X-7, and \#X-8, respectively. ***p $<0.001 ; * * p 0.01 ; * p<0.05$.

total of 33 samples for SARS-CoV-2 spike protein and 33 samples for nucleocapsid protein tests are prepared.

As shown in Figure 2a, the diluted IPG30 MNPs (particle concentration of $3.06 \mathrm{nM}$ ) are incubated with spike or nucleocapsid pAbs for $12 \mathrm{~h}$ at $4{ }^{\circ} \mathrm{C}$ on an incubating shaker. This step allows the $\mathrm{Fc}$ part of IgG pAbs to bind to protein G from IPG30 MNP surfaces. Then, $80 \mu \mathrm{L}$ of pAb-functionalized MNPs is mixed with $80 \mu \mathrm{L}$ of spike or nucleocapsid proteins and incubated at room temperature for $1 \mathrm{~h}$ with continuous shaking. After this point, the specific binding between pAbs and target protein molecules reaches to an equilibrium state, and sample vials are then stored at $4{ }^{\circ} \mathrm{C}$ before MPS tests. It should be noted that the $1 \mathrm{~h}$ incubation time of pAb-functionalized MNPs with protein biomarkers is not optimized. To ensure the antibody-antigen binding to fully saturate, we set an incubation time of $1 \mathrm{~h}$. Further works should be carried out to reduce the incubation time by means of changing incubation conditions (such as temperature, ${ }^{49,50}$ pressure, ${ }^{51}$ flow rate, ${ }^{52}$ etc.) or choosing higher binding affinity pAbs. ${ }^{53}$

\section{RESULTS AND DISCUSSION}

3.1. Time- and Frequency-Domain Signals Recorded by One-Stage Lock-In MPS System. The real-time voltage signals of the blank sample (no IPG30 MNPs loaded, i.e., noise floor), bare MNPs (vial \#9, IPG30 MNPs without surface functionalization), vial \#IV-8 $(\mathrm{MNP} / \mathrm{pAb}=1: 1)$, and vial \#I-8 $(\mathrm{MNP} / \mathrm{pAb}=1: 4)$ as recorded from pick-up coils are plotted in Figure 3a. The background signal from the blank sample is a relatively smooth sinusoidal waveform, while all samples with MNPs loaded show visible spikes. These spikes are caused by the dynamic magnetic responses of MNPs in the liquid phase under external AC magnetic fields (excitation fields), as explained in Section 2.4. The bare MNPs cause relatively stronger spikes (red curve) than the MNPs functionalized with one pAb each (blue curve, $\mathrm{MNP} / \mathrm{pAb}=1: 1$, vial \#IV-8), and the MNPs functionalized with four pAbs each (magenta curve, $\mathrm{MNP} / \mathrm{pAb}=1: 4$, vial \#I-8) show the weakest spikes. A zoomed-in view of these spikes is presented in Figure 3a, and the amplitudes of these spikes are marked in dashed lines. The frequency domain MPS spectra are obtained by carrying out DFT on the time domain voltage signal. As shown in Figure 3c, the bare MNP sample shows highest harmonic amplitudes, followed by $\mathrm{MNP} / \mathrm{pAb}=1: 1$ (vial \#IV-8) and $\mathrm{MNP} / \mathrm{pAb}=$ 1:4 (vial \#I-8), and the blank samples show the weakest and negligible harmonics. The results confirm that conjugating 
pAbs on MNP surfaces does hinder the physical rotational motion of MNPs and cause weaker harmonics. In addition, based on the extent of harmonic signal drop (compared with bare MNP sample) from $\mathrm{MNP} / \mathrm{pAb}=1: 1$ and $\mathrm{MNP} / \mathrm{pAb}=$ $1: 4$, we can confirm that the pAbs are successfully functionalized onto MNPs. The functionalization of different amounts of pAbs on MNPs is further confirmed by the hydrodynamic sizes (see Supporting Information S3). In addition, the colloidal stability of MNPs after coating pAbs is confirmed by zeta potential results, as summarized in Supporting Information S3.

3.2. Concentration-Response Profiles of SARS-CoV-2 Spike Protein. Six independent MPS readings are taken from each of the 33 samples and the 3rd harmonics from 32 samples (Group I-IV) are summarized in Figure $4 \mathrm{a}-\mathrm{d}$. Two-sample $t$ test is applied to compare the differences between two sets of harmonic data collected from different samples.

For the scenario of coating four pAbs onto each MNP (Group I, $\mathrm{MNP} / \mathrm{pAb}=1: 4$, Figure $4 \mathrm{a}$ ), adding $400 \mathrm{nM}$ (equivalent to 32 pmole) of spike protein to vial \#I-1 causes the 3rd harmonic to be significantly lower $(p<0.001)$ than the 3rd harmonics from other vials in Group I. This weaker harmonic signal is due to the abundancy of target spike protein molecules from the sample that causes the nanoparticle clustering, as explained in Section 2.4 and Figure 2c,d. However, vials \#I-2 to \#I-8 show no significant differences.

In Group II ( $\mathrm{MNP} / \mathrm{pAb}=1: 3$, Figure $4 \mathrm{~b})$, vials \#II-1 to \#II4 show similar harmonic amplitudes and are significantly lower than other vials from the same group, which indicates that in this scenario $(\mathrm{MNP} / \mathrm{pAb}=1: 3)$, adding 32 pmole $(400 \mathrm{nM}), 8$ pmole (100 nM), 2 pmole $(25 \mathrm{nM})$, and 1 pmole $(12.5 \mathrm{nM})$ SARS-CoV-2 spike protein can completely block the Brownian relaxation of MNPs and cause the lowest achievable MPS spectra (or weakest dynamic magnetic responses). By reducing the number of spike protein in vials \#II-5 to \#II-8, the 3rd harmonic amplitudes gradually increase due to the less amount of the target spike protein molecule present in the sample that can cause the nanoparticle clustering. From Group II, a clear linear concentration-response curve is observed from vials \#II4 to \#II-8. Indicating that this bioassay design could be used for quantitative assays of spike protein samples within a concentration range of $0-12.5 \mathrm{nM}$ (corresponding to the spike protein amount of $0-1$ pmole) and qualitative assays of spike protein samples with concentrations above $12.5 \mathrm{nM}$.

On the other hand, by functionalizing two pAbs (Group III, $\mathrm{MNP} / \mathrm{pAb}=1: 2$, Figure $4 \mathrm{c}$ ) and one pAb (Group IV, MNP/ $\mathrm{pAb}=1: 1$, Figure $4 \mathrm{~d}$ ) per MNP, no significant response curves are observed. There is a clear trend showing that the harmonic signal amplitude decreases, as we add a higher number of spike protein molecules.

Figure 4e compares the 3rd harmonic amplitudes from vials \#X-1 (X = I, II, III, and IV), bare MNP (vial \#9), and blank (no IPG30 MNP, i.e., noise floor). All the vials added with IPG30 MNPs show significantly higher harmonic signal intensities than the blank sample, of which the bare MNP (vial \#9) sample shows the highest harmonic signal because no pAbs are functionalized and the Brownian relaxation of MNPs is not hindered even by adding $400 \mathrm{nM}$ (32 pmole) spike protein to it, which confirms that MNPs without pAb functionalization will not form clusters in the presence of target analytes. In addition, the 3rd harmonics from vials \#X-3, \#X-5, \#X-7, and \#X-8 (X = I, II, III, and IV) are compared in Figure $4 \mathrm{f}-\mathrm{i}$, respectively. A clear trend is observed that by adding the same amount of spike protein molecules, MNPs that functionalized with more pAbs show a lower harmonic signal. Figure $4 \mathrm{i}$ proves the schematic drawing in Figure $2 \mathrm{~b}$ that functionalizing more pAbs per MNP will cause weaker dynamic magnetic responses and lower harmonic signals, which also confirms that the pAbs have been successfully functionalized onto IPG30 MNPs.

From the concentration-response profiles of SARS-CoV-2 spike protein, it is concluded that functionalizing three pAbs per MNP (Group II, MNP/pAb = 1:3, Figure $4 \mathrm{~b}$ ) gives us the best linear response curve with a detection limit of $1.56 \mathrm{nM}$ (equivalent to 125 fmole of spike protein molecules).

In Figure 5, we have summarized the ratios of higher harmonics (from the 5th to the 15th harmonics) to the 3rd

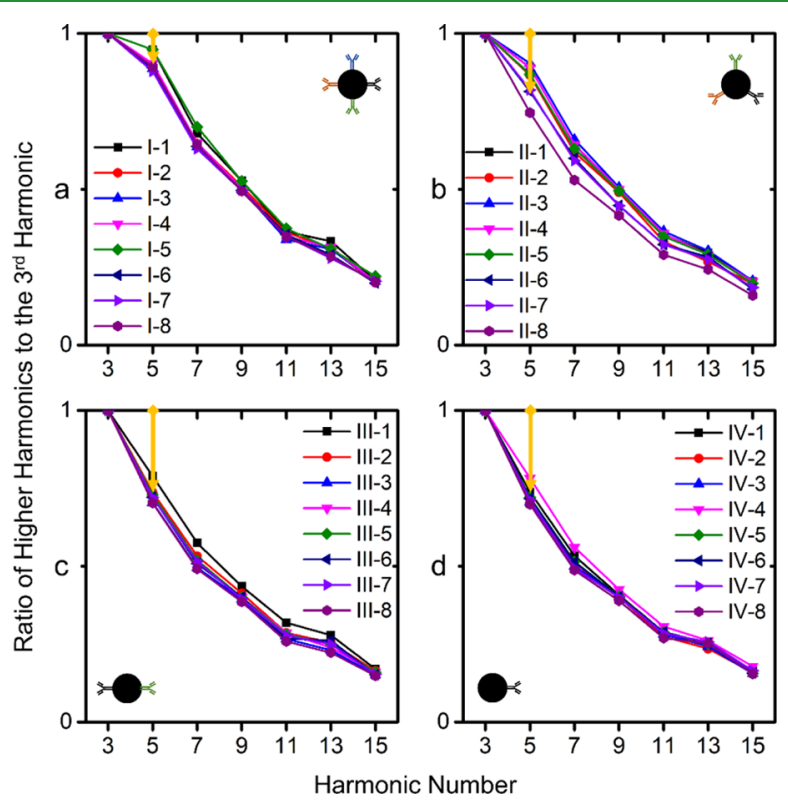

Figure 5. Ratios of higher harmonics to the 3rd harmonics recorded from Groups (a) I, (b) II, (c) III, and (d) IV. Yellow double arrows mark the decay rate from the 5th to the 3rd harmonics across Groups I to IV.

harmonics collected from each sample. For Groups I, III, and IV (Figure 5a,c,d), the harmonic ratio curves of vials \#X-1 to \#X-8 (X = I, III, and IV) are tightly distributed with very narrow gaps or even overlapping, while the harmonic ratio curves from Group II vials \#II-1 to \#II-8 are sparsely distributed. The significant differences in harmonic ratio curves from vials added with different concentrations/amounts of spike protein molecules allow us to analyze and collect meaningful concentration-response profiles, as shown in Figure $4 \mathrm{~b}$.

The harmonic amplitude decreases as the harmonic index increases, namely, the harmonic amplitudes A3 > A5 > A7 > A9 $>$ A11 $>$ A13 > A15, where $A$ represents amplitude (A). It is observed that functionalizing different amount of pAbs per MNP also changes the decay rate of higher harmonics (with a harmonic index), as reflected in the MPS spectra from Figure $3 c$. The yellow double arrows shown in Figure 5a-d mark the decay rates from the 5 th to the 3rd harmonics across Groups I to IV. In average, the R53 values (harmonic amplitude ratio of A5 over the A3) from Groups I-IV are 0.91, 0.85, 0.73, and 0.72 , respectively. Also, the R73 values (harmonic amplitude ratio of the A7 over the A3) from Groups I-IV are 0.65, 0.61, 

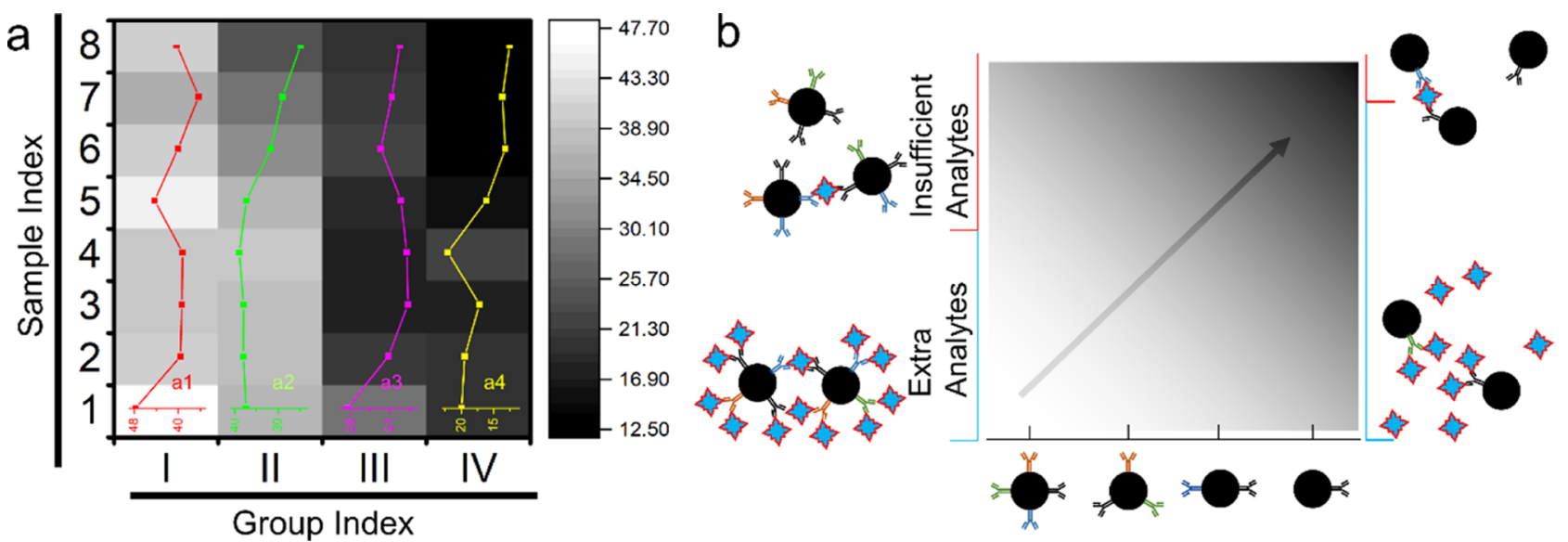

Figure 6. (a) Grayscale heatmaps of the 3rd harmonic signal drop $\Delta$ (in \%) compared to bare MNPs for SARS-CoV-2 spike proteins. A total of 32 samples in groups I-IV are compared with the 3rd harmonic from bare MNPs (vial \#9). [a(1-4)] are the harmonic signal drop, $\Delta$, plotted as a function of spike protein amount/concentration for Groups I-IV, respectively. (b) Grayscale heatmap showing the ideal color trend regarding different amounts of pAbs functionalized on MNPs and different scenarios of extra and insufficient target analytes (i.e., SARS-CoV-2 spike protein).

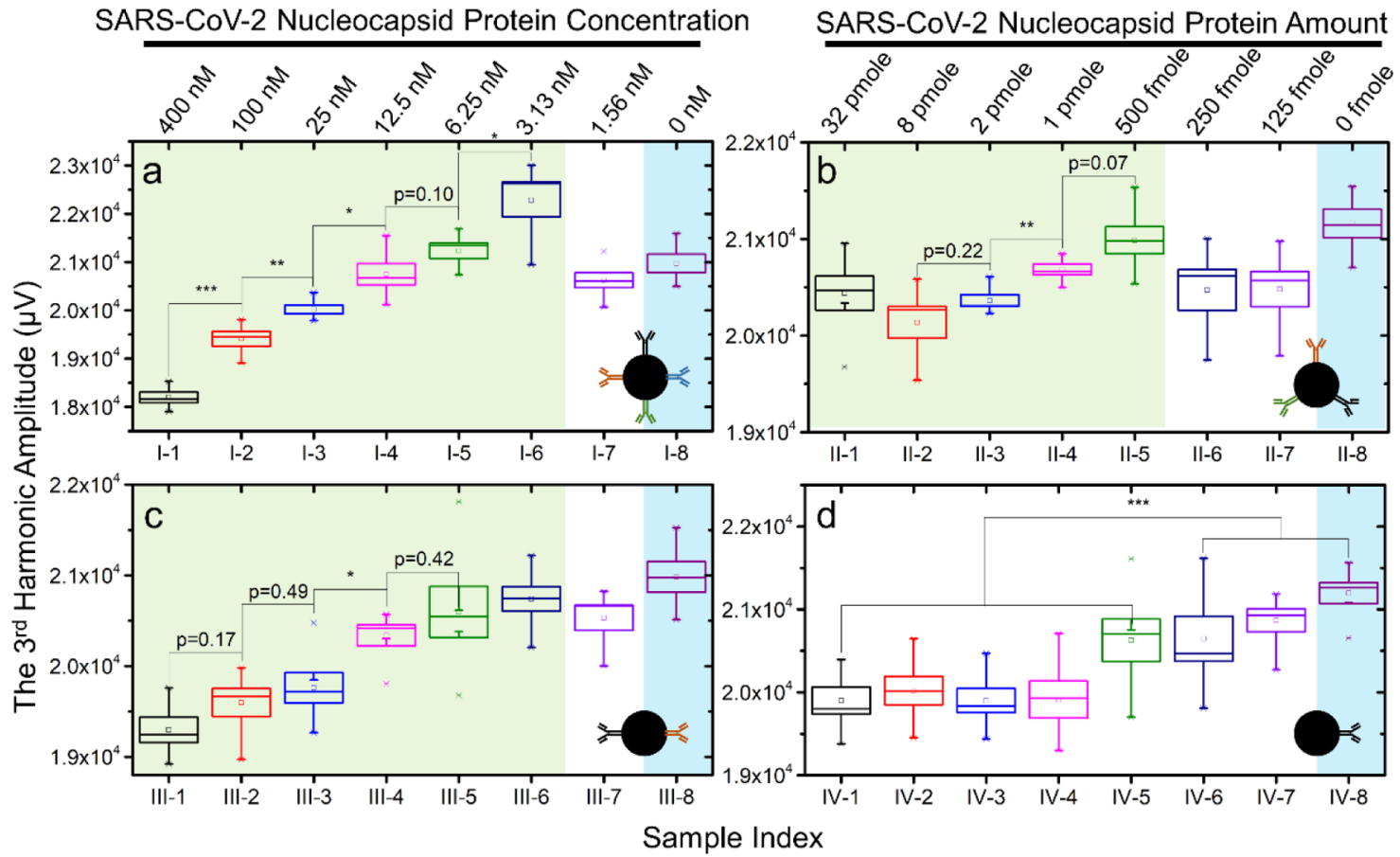

Figure 7. Concentration-response profiles of the SARS-CoV-2 nucleocapsid protein. (a-d) Box plots of the 3rd harmonic amplitudes from vials \#X-1 to \#X-8, where $\mathrm{X}=\mathrm{I}$, II, III, and IV, respectively. The highlighted green areas are the monotonic concentration-response regions, and the highlighted blue areas are the vials \#X-8, namely, control samples without the addition of target analytes. Middle horizontal lines represent the average signal, and top and bottom horizontal lines represent standard errors. $* * * p<0.001 ; * * p<0.01 ; *<0.05$.

0.52 , and 0.51 , respectively. Thus, conjugating more pAbs on each MNP will make the higher harmonics decay slower.

Besides comparing each sample's higher harmonic amplitudes (A5-A15) to its own 3rd harmonic (A3), we further compared the samples' higher harmonics from Group I-IV with the bare MNP sample (vial \#9, IPG30 MNP without any surface functionalization). As shown in Supporting Information S4, the 3rd, 5th, 7th, 9th, 11th, 13th, and 15th harmonics of each sample are compared with the corresponding harmonics from the bare MNP sample (vial \#9) and the grayscale heatmaps of the harmonic signal drop (defined as $\Delta=\frac{A i_{9}-A i_{X-j}}{A i_{9}} \times 100 \%$, where $i$ is the harmonic index, the subscripts are sample indexes, $\mathrm{X}=\mathrm{I}, \mathrm{II}, \mathrm{III}$, and IV, and $j=1,2$,
$3, \ldots, 8)$ are plotted. Figure 6a compares the 3rd harmonic of each sample with the bare MNP sample (vial \#9). In each row of Figure 6a, by adding the same amount of spike protein molecules, the 3 rd harmonic signal drop $\Delta$ decreases from I to IV, which agrees with the results shown in Figure $4 \mathrm{e}-\mathrm{i}$. Thus, ideally, the color becomes darker from the left column to right column in each grayscale heatmap (as schemed in Figure 6b). In each column of Figure 6a, with IPG30 MNPs surface functionalized with an identical number of pAbs, adding more spike protein causes a larger harmonic signal drop. Ideally, the color becomes darker from the bottom row (vials \#X-1, X = I, II, III, and IV) to the top row (vials \#X-8, X = I, II, III, and IV). Again, this ideal trend is shown in Figure $6 \mathrm{~b}$. 
There are two variables in plotting the grayscale heatmaps of the higher harmonic signal drop $\Delta:$ (1) the number of pAbs functionalized on each MNP (Groups I-IV with MNP $/ \mathrm{pAb}=$ $1: 4,1: 3,1: 2$, and $1: 1$ ) and (2) the concentration/amount of the spike protein added to each sample (i.e., vials \#X-1 to \#X$8, \mathrm{X}=\mathrm{I}$, II, III, and IV). These two variables jointly cause the harmonic signal drop $\Delta$ increases from the upper right corner to bottom left corner in diagonal. The 3rd harmonics give a highest signal to noise ratio, and we can clearly see this trend from Figure 6a. Figure 6a(1-4) plots the 3rd harmonic signal drop $\Delta$ curves for samples from Groups I-IV, where Group II shows the best monotonic concentration-response curve. Figure $6 \mathrm{~b}$ schematically shows the scenarios where extra and insufficient target analytes (i.e., spike protein) are added. As a result, the number of target analytes directly affects the degree of nanoparticle clustering and the dynamic magnetic responses.

3.3. Concentration-Response Profiles of SARS-CoV-2 Nucleocapsid Protein. A total of 33 SARS-CoV-2 nucleocapsid samples are prepared in the same manner as the spike protein samples aforementioned. Six independent MPS readings are taken from each sample, and the $3 \mathrm{rd}$ harmonics are plotted in Figure $7 \mathrm{a}-\mathrm{d}$. Group I $(\mathrm{MNP} / \mathrm{pAb}=$ 1:4) shows a remarkable monotonic concentration-response curve where the 3 rd harmonics monotonically increase from vial \#I-1 to vial \#I-6, with nucleocapsid protein decreasing from 400 ( 32 pmole) to $3.13 \mathrm{nM}$ (500 fmole). Similar trends are also observed from Group II $(\mathrm{MNP} / \mathrm{pAb}=1: 3$, Figure $7 \mathrm{~b})$ and Group III $(\mathrm{MNP} / \mathrm{pAb}=1: 2$, Figure $7 \mathrm{c})$, while both are less significant than Group I. The detection limit of the SARSCoV-2 nucleocapsid protein on our MPS system is $12.5 \mathrm{nM}$ (equivalent to 1 pmole) based on the response curves from Group I.

We also observed that when functionalizing one pAb per MNP (Group IV), no remarkable response curves are observed from either spike or nucleocapsid proteins (Figures $4 \mathrm{~d}$ and $7 \mathrm{~d})$. For Group IV $(\mathrm{MNP} / \mathrm{pAb}=1: 1)$ in spike protein tests, vials \#IV-1 to \#IV-4 are significantly different $(p<0.001)$ from vials \#IV-5 to \#IV-8, but no significant differences are observed within vials \#IV-1 to \#IV-4 or vials \#IV-5 to \#IV-8, as shown in Figure 4d. Similar results are observed from Group IV (MNP/ $\mathrm{pAb}=1: 1)$ in nucleocapsid protein tests, and vials \#IV-1 to \#IV-5 are significantly different $(p<0.001)$ from vials \#IV-6 to \#IV-8. Thus, for the detection of spike and nucleocapsid proteins, it is not practical to functionalize one pAb per MNP. The grayscale heatmaps of the harmonic signal drop $\Delta$ from nucleocapsid samples are also provided in Supporting Information S5. The ratios of higher harmonics to the 3rd harmonics recorded from Groups I to IV for the nucleocapsid protein can be found in Supporting Information S6.

\section{CONCLUSIONS}

Herein, we have successfully applied a one-stage lock-in MPS system for the detection of SARS-CoV-2 spike and nucleocapsid proteins directly in the liquid phase. This onestep, wash-free detection method allows a layperson to carry out the tests without much technical training requirements. By conjugating pAbs to MNPs, it increases the chances of MNP binding events to target protein molecules (i.e., spike and nucleocapsid proteins in this work). Combined with the nature that each protein molecule has different epitopes that allow for the binding of multiple pAbs. We are able to amplify the effect of MNP binding events that caused obstruction of physical rotational motion of MNPs, thus amplifying the changes in higher harmonics.

In addition, we explored the effect of the pAbs amount functionalized on each MNP. Four groups of samples with $\mathrm{MNP} / \mathrm{pAb}=1: 4$ (Group I), 1:3 (Group II), 1:2 (Group III), and 1:1 (Group IV) are designed. These pAb-functionalized MNPs are used to quantitatively detect spike and nucleocapsid proteins varying from 0 fmole (vials \#X-8) to 32 pmole (vials $\# \mathrm{X}-1$ ). The concentration-response profiles for spike and nucleocapsid proteins are generated based on the higher harmonics from MNPs. It is concluded that conjugating three pAbs per MNP yields a linear concentration-response curve and reaches a detection limit of $1.56 \mathrm{nM}$ (equivalent to 125 fmole) for spike protein molecules. On the other hand, conjugating four pAb per MNP yields a linear concentrationresponse curve for the detection of nucleocapsid proteins and a detection limit of $12.5 \mathrm{nM}$ (equivalent to 1 pmole) is achieved. The higher harmonics from all the control groups, bare MNPs (vial \#9) and vials \#X-8 (X = I, II, III, and IV, where no proteins are added), confirmed that the pAbs have been successfully conjugated to MNPs.

To sum up, this nanoparticle clustering-based bioassay mechanism combined with the MPS platform is intrinsically versatile and can be applied for the detections of other biomarkers such as proteins, viruses, nucleic acids, and so forth. Its nature of one-step wash-free experimental procedure allows for simpler and more convenient on-field tests in the future. Zhong et al. also reported the SARS-CoV-2 spike protein detection from the liquid phase using a similar MPS platform, ${ }^{23}$ where they coated anti-SARS-CoV-2 spike protein monoclonal antibodies (mAbs) to the MNPs and spike protein to $100 \mathrm{~nm}$ polystyrene (PS) beads. The binding events of MNPs to $100 \mathrm{~nm}$ PS beads through antibody-antigen-specific recognition hinder the physical rotational motion of MNPs. Thus, in a similar manner, higher harmonics are recorded as indicators of spike protein abundancy. Each PS bead was coated with 100 SARS-CoV-2 spike proteins to mimic one SARS-CoV-2 virus particle. They demonstrated the detection limit of $84 \mathrm{fM}$ (equal to $5.9 \mathrm{fmole}$ ) mimic virus particles, which is equal to 590 fmole of spike protein molecules. The main differences between our work and Zhong et al.'s work are that we utilized MNP-pAb-protein-pAb-MNP-pAb-protein-pAbMNP-... clustering that caused obstruction of physical rotational motion of MNPs to achieve specific detection of the target SARS-CoV-2 spike and nucleocapsid protein, while Zhong et al. used an MNP-mAb-protein-PS bead to block the physical rotational motion of MNPs. Although the detection strategy is different, the detection mechanism is the same, that is, relying on the change of the harmonic signal caused by the reduced rotational freedom of the MNPs to quantitatively and/ or qualitatively detect the target the SARS-CoV-2 protein biomarker.

It should also be noted that the results presented in this work are based on detecting purified spike and nucleocapsid proteins from PBS buffer. This is a feasibility demonstration of the one-stage lock-in MPS system for protein biomarker detection, and we did not measure SARS-CoV-2 biomarkers from clinical samples such as upper respiratory tract mucus including saliva and sputum. There are several factors that may impair the sensitivity and repeatability of MPS platforms when testing clinical samples, such as viscosity of biofluid samples, temperature, and nonspecific binding of unknown chemical compounds from biofluids. Because this one-step liquid phase 
detection mechanism relies on the binding event-caused Brownian relaxation change in MNPs, the viscosity and temperature of biofluid samples can cause different degrees of deviations in MPS harmonic signals and the nonspecific binding can cause repeatability issues (as well as false positive results). To avoid the viscosity variation caused by MPS signal bias, several sample preparation and collection methods can be adopted, such as using the exhaled breath condensate that contains respiratory droplets from the lower respiratory tract. $^{54-56}$ The condensed water droplets containing SARS$\mathrm{CoV}-2$ virions can effectively avoid the body fluid (i.e., sputum and saliva) that caused viscosity variations between patients. In addition to the sample collection method, the sample preparation method can also be altered for this specific application. Usually, the upper respiratory tract mucus samples (nasopharyngeal swabs, throat swabs, nasopharyngeal wash, saliva, sputum, etc.) have high virus titer, but the complex internal matrix could interfere with the MPS test results due to the high viscosity and unknown chemical compounds that nonspecifically bind to MNPs. ${ }^{57,58}$ The binding specificities of pAbs used in this work have been confirmed by ELISA testing (see Supporting Information S7). A mature sample preparation process has been applied to dilute specimens by washing buffers and filter out larger particle complexes. ${ }^{59-61}$ To avoid the nonspecific binding of unknown chemical compounds from the complex matrix, surface-functionalized MNPs can be PEGylated, ${ }^{62-64}$ where polyethylene glycol is a hydrophilic and neutrally charged polymer that can help prevent the nonspecific bindings of other chemical compounds to MNP surfaces.

\section{ASSOCIATED CONTENT}

\section{(s) Supporting Information}

The Supporting Information is available free of charge at https://pubs.acs.org/doi/10.1021/acsami.1c14657.

Static magnetic hysteresis loops and magnetic properties of IPG30 MNPs; minimum detectable amount of IPG30 by the homebuilt MPS system; hydrodynamic size, zeta potential, and the 3rd harmonic amplitudes of bare MNPs and MNPs functionalized with different amounts of pAbs; grayscale heatmaps of the higher harmonic signal drop $\Delta$ (in \%) compared to bare MNPs for SARSCoV-2 spike proteins; grayscale heatmaps of the higher harmonic signal drop $n$ (in \%) compared to bare MNPs for SARS-CoV-2 nucleocapsid proteins; ratios of higher harmonics to the 3rd harmonics recorded from Groups I to IV for the SARS-CoV-2 nucleocapsid protein; and specificity of the SARS-CoV-2 spike and nucleocapsid pAbs confirmed by ELISA (PDF)

\section{AUTHOR INFORMATION}

\section{Corresponding Authors}

Kai Wu - Department of Electrical and Computer Engineering, University of Minnesota, Minneapolis, Minnesota 55455, United States; (1) orcid.org/0000-0002-9444-6112; Email: wuxx0803@umn.edu

Maxim C-J Cheeran - Department of Veterinary Population Medicine, University of Minnesota, St. Paul, Minnesota 55108, United States; Email: cheeran@umn.edu

Jian-Ping Wang - Department of Electrical and Computer Engineering, University of Minnesota, Minneapolis, Minnesota 55455, United States; Email: jpwang@umn.edu

\section{Authors}

Vinit Kumar Chugh - Department of Electrical and Computer Engineering, University of Minnesota, Minneapolis, Minnesota 55455, United States; 이이이.org/0000-0001$7818-7811$

Venkatramana D. Krishna - Department of Veterinary Population Medicine, University of Minnesota, St. Paul,

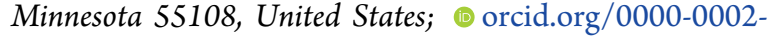
1980-5525

Arturo di Girolamo - Department of Electrical and Computer Engineering, University of Minnesota, Minneapolis, Minnesota 55455, United States

Yongqiang Andrew Wang - Ocean Nano Tech LLC, San Diego, California 92126, United States

Renata Saha - Department of Electrical and Computer Engineering, University of Minnesota, Minneapolis, Minnesota 55455, United States; (1) orcid.org/0000-00020389-0083

Shuang Liang - Department of Chemical Engineering and Material Science, University of Minnesota, Minneapolis, Minnesota 55455, United States; 이이.org/0000-0003$1491-2839$

Complete contact information is available at: https://pubs.acs.org/10.1021/acsami.1c14657

\section{Author Contributions}

${ }^{\perp}$ K.W., V.K.C., and V.D.K. have contributed equally to this work. K.W., M.C-J.C., and J.-P.W conceived the experiment. K.W. carried out part of the MPS-based tests, plotted the figures, and wrote part of the paper. V.K.C. carried out part of the MPS-based tests, designed the circuitry and assembled the circuit boards, and wrote part of the paper. V.D.K. prepared all the experimental samples and wrote part of the paper. A.G. assisted in the design of circuitry and assemble of circuit boards. Y.A.W. synthesized the MNPs. R.S. assisted in MPS raw data processing and the data collection of hydrodynamic size and zeta potential. S.L. assisted in MPS raw data processing and analysis. All authors proofread the paper.

\section{Notes}

The authors declare no competing financial interest.

\section{ACKNOWLEDGMENTS}

This study was financially supported by the Institute of Engineering in Medicine, the Robert F. Hartmann Endowed Chair professorship, the University of Minnesota Medical School, and the University of Minnesota Physicians and Fairview Health Services through COVID-19 Rapid Response Grant. This study was also financially supported by the U.S. Department of Agriculture-National Institute of Food and Agriculture (NIFA) under award number 2020-67021-31956. Research reported in this publication was supported by the National Institute Of Dental \& Craniofacial Research of the National Institutes of Health under award number R42DE030832. The content is solely the responsibility of the authors and does not necessarily represent the official views of the National Institutes of Health. Portions of this work were conducted in the Minnesota Nano Center, which is supported by the National Science Foundation through the National Nano Coordinated Infrastructure Network (NNCI) under award number ECCS-1542202. 


\section{REFERENCES}

(1) Zhou, P.; Yang, X.-L.; Wang, X.-G.; Hu, B.; Zhang, L.; Zhang, W.; Si, H.-R.; Zhu, Y.; Li, B.; Huang, C.-L.; Chen, H.-D.; Chen, J.; Luo, Y.; Guo, H.; Jiang, R.-D.; Liu, M.-Q.; Chen, Y.; Shen, X.-R.; Wang, X.; Zheng, X.-S.; Zhao, K.; Chen, Q.-J.; Deng, F.; Liu, L.-L.; Yan, B.; Zhan, F.-X.; Wang, Y.-Y.; Xiao, G.-F.; Shi, Z.-L. A Pneumonia Outbreak Associated with a New Coronavirus of Probable Bat Origin. Nature 2020, 579, 270-273.

(2) Huang, C.; Wang, Y.; Li, X.; Ren, L.; Zhao, J.; Hu, Y.; Zhang, L.; Fan, G.; Xu, J.; Gu, X.; Cheng, Z.; Yu, T.; Xia, J.; Wei, Y.; Wu, W.; Xie, X.; Yin, W.; Li, H.; Liu, M.; Xiao, Y.; Gao, H.; Guo, L.; Xie, J.; Wang, G.; Jiang, R.; Gao, Z.; Jin, Q.; Wang, J.; Cao, B. Clinical Features of Patients Infected with 2019 Novel Coronavirus in Wuhan, China. Lancet 2020, 395, 497-506.

(3) Lu, R.; Zhao, X.; Li, J.; Niu, P.; Yang, B.; Wu, H.; Wang, W.; Song, H.; Huang, B.; Zhu, N.; Bi, Y.; Ma, X.; Zhan, F.; Wang, L.; Hu, T.; Zhou, H.; Hu, Z.; Zhou, W.; Zhao, L.; Chen, J.; Meng, Y.; Wang, J.; Lin, Y.; Yuan, J.; Xie, Z.; Ma, J.; Liu, W. J.; Wang, D.; Xu, W.; Holmes, E. C.; Gao, G. F.; Wu, G.; Chen, W.; Shi, W.; Tan, W. Genomic Characterisation and Epidemiology of 2019 Novel Coronavirus: Implications for Virus Origins and Receptor Binding. Lancet 2020, 395, 565-574.

(4) Chan, J. F.-W.; Kok, K.-H.; Zhu, Z.; Chu, H.; To, K. K.-W.; Yuan, S.; Yuen, K.-Y. Genomic Characterization of the 2019 Novel Human-Pathogenic Coronavirus Isolated from a Patient with Atypical Pneumonia after Visiting Wuhan. Emerging Microbes Infect. 2020, 9, 221-236.

(5) Wu, F.; Zhao, S.; Yu, B.; Chen, Y.-M.; Wang, W.; Song, Z.-G.; Hu, Y.; Tao, Z.-W.; Tian, J.-H.; Pei, Y.-Y.; Yuan, M.-L.; Zhang, Y.-L.; Dai, F.-H.; Liu, Y.; Wang, Q.-M.; Zheng, J.-J.; Xu, L.; Holmes, E. C.; Zhang, Y.-Z. A New Coronavirus Associated with Human Respiratory Disease in China. Nature 2020, 579, 265-269.

(6) Zhu, N.; Zhang, D.; Wang, W.; Li, X.; Yang, B.; Song, J.; Zhao, X.; Huang, B.; Shi, W.; Lu, R. A Novel Coronavirus from Patients with Pneumonia in China, 2019. N. Engl. J. Med. 2020, 382, 727-733.

(7) Safiabadi Tali, S. H.; LeBlanc, J. J.; Sadiq, Z.; Oyewunmi, O. D.; Camargo, C.; Nikpour, B.; Armanfard, N.; Sagan, S. M.; JahanshahiAnbuhi, S. Tools and Techniques for Severe Acute Respiratory Syndrome Coronavirus 2 (SARS-CoV-2)/COVID-19 Detection. Clin. Microbiol. Rev. 2021, 34, No. e00228.

(8) Younes, N.; Al-Sadeq, D. W.; Al-Jighefee, H.; Younes, S.; AlJamal, O.; Daas, H. I.; Yassine, H. M.; Nasrallah, G. K. Challenges in Laboratory Diagnosis of the Novel Coronavirus SARS-CoV-2. Viruses 2020, 12, 582.

(9) Yüce, M.; Filiztekin, E.; Özkaya, K. G. COVID-19 Diagnosis-A Review of Current Methods. Biosens. Bioelectron. 2020, 172, 112752.

(10) Chu, D. K. W.; Pan, Y.; Cheng, S. M. S.; Hui, K. P. Y.; Krishnan, P.; Liu, Y.; Ng, D. Y. M.; Wan, C. K. C.; Yang, P.; Wang, Q.; Peiris, M.; Poon, L. L. M. Molecular Diagnosis of a Novel Coronavirus (2019-NCoV) Causing an Outbreak of Pneumonia. Clin. Chem. 2020, 66, 549-555.

(11) Loeffelholz, M. J.; Tang, Y.-W. Laboratory Diagnosis of Emerging Human Coronavirus Infections-the State of the Art. Emerging Microbes Infect. 2020, 9, 747-756.

(12) Porte, L.; Legarraga, P.; Vollrath, V.; Aguilera, X.; Munita, J. M.; Araos, R.; Pizarro, G.; Vial, P.; Iruretagoyena, M.; Dittrich, S.; Weitzel, T. Evaluation of a Novel Antigen-Based Rapid Detection Test for the Diagnosis of SARS-CoV-2 in Respiratory Samples. Int. J. Infect. Dis. 2020, 99, 328-333.

(13) Mavrikou, S.; Moschopoulou, G.; Tsekouras, V.; Kintzios, S. Development of a Portable, Ultra-Rapid and Ultra-Sensitive CellBased Biosensor for the Direct Detection of the SARS-CoV-2 S1 Spike Protein Antigen. Sensors 2020, 20, 3121.

(14) Lin, Q.; Wen, D.; Wu, J.; Liu, L.; Wu, W.; Fang, X.; Kong, J. Microfluidic Immunoassays for Sensitive and Simultaneous Detection of IgG/IgM/Antigen of SARS-CoV-2 within 15 Min. Anal. Chem. 2020, 92, 9454-9458.

(15) Mak, G. C.; Cheng, P. K.; Lau, S. S.; Wong, K. K.; Lau, C.; Lam, E. T.; Chan, R. C.; Tsang, D. N. Evaluation of Rapid Antigen
Test for Detection of SARS-CoV-2 Virus. J. Clin. Virol. 2020, 129, 104500.

(16) Wu, K.; Su, D.; Saha, R.; Liu, J.; Chugh, V. K.; Wang, J.-P. Magnetic Particle Spectroscopy: A Short Review of Applications Using Magnetic Nanoparticles. ACS Appl. Nano Mater. 2020, 3, 4972-4989.

(17) Nikitin, P. I.; Vetoshko, P. M.; Ksenevich, T. I. New Type of Biosensor Based on Magnetic Nanoparticle Detection. J. Magn. Magn. Mater. 2007, 311, 445-449.

(18) Wu, K.; Su, D.; Saha, R.; Wong, D.; Wang, J.-P. Magnetic Particle Spectroscopy-Based Bioassays: Methods, Applications, Advances, and Future Opportunities. J. Phys. Appl. Phys. 2019, 52, 173001.

(19) Krause, H.-J.; Wolters, N.; Zhang, Y.; Offenhäusser, A.; Miethe, P.; Meyer, M. H. F.; Hartmann, M.; Keusgen, M. Magnetic Particle Detection by Frequency Mixing for Immunoassay Applications. J. Magn. Magn. Mater. 2007, 311, 436-444.

(20) Orlov, A. V.; Khodakova, J. A.; Nikitin, M. P.; Shepelyakovskaya, A. O.; Brovko, F. A.; Laman, A. G.; Grishin, E. V.; Nikitin, P. I. Magnetic Immunoassay for Detection of Staphylococcal Toxins in Complex Media. Anal. Chem. 2013, 85, 11541163.

(21) Orlov, A. V.; Znoyko, S. L.; Cherkasov, V. R.; Nikitin, M. P.; Nikitin, P. I. Multiplex Biosensing Based on Highly Sensitive Magnetic Nanolabel Quantification: Rapid Detection of Botulinum Neurotoxins A, B, and E in Liquids. Anal. Chem. 2016, 88, 1041910426.

(22) Wu, K.; Chugh, V. K.; Di Girolamo, A.; Liu, J.; Saha, R.; Su, D.; Krishna, V. D.; Nair, A.; Davies, W.; Wang, Y. A.; Cheeran, M. C.-J.; Wang, J.-P. A Portable Magnetic Particle Spectrometer for Future Rapid and Wash-Free Bioassays. ACS Appl. Mater. Interfaces 2021, 13, $7966-7976$.

(23) Zhong, J.; Rösch, E. L.; Viereck, T.; Schilling, M.; Ludwig, F. Toward Rapid and Sensitive Detection of SARS-CoV-2 with Functionalized Magnetic Nanoparticles. ACS Sens. 2021, 6, 976-984.

(24) Zhong, J.; Janssen, K.-J.; Draack, S.; Viereck, T.; Schilling, M.; Ludwig, F. Dependence of Biomolecule Detection on Magnetic Nanoparticle Concentration. J. Magn. Magn. Mater. 2021, 517, 167408.

(25) Wu, K.; Schliep, K.; Zhang, X.; Liu, J.; Ma, B.; Wang, J. P. Characterizing Physical Properties of Superparamagnetic Nanoparticles in Liquid Phase Using Brownian Relaxation. Small 2017, 13,1604135 .

(26) Wu, K.; Liu, J.; Su, D.; Saha, R.; Wang, J.-P. Magnetic Nanoparticle Relaxation Dynamics-Based Magnetic Particle Spectroscopy for Rapid and Wash-Free Molecular Sensing. ACS Appl. Mater. Interfaces 2019, 11, 22979-22986.

(27) Gordon-Wylie, S. W.; Ness, D. B.; Shi, Y.; Mirza, S. K.; Paulsen, K. D.; Weaver, J. B. Measuring Protein Biomarker Concentrations Using Antibody Tagged Magnetic Nanoparticles. Biomed. Phys. Eng. Express 2020, 6, 065025.

(28) Gordon-Wylie, S. W.; Grüttner, C.; Teller, H.; Weaver, J. B. Using Magnetic Nanoparticles and Protein-Protein Interactions to Measure $\mathrm{pH}$ at the Nanoscale. IEEE Sens. Lett. 2020, 4, 1-3.

(29) Shi, Y.; Jyoti, D.; Gordon-Wylie, S. W.; Weaver, J. B. Quantification of Magnetic Nanoparticles by Compensating for Multiple Environment Changes Simultaneously. Nanoscale 2020, 12, 195-200.

(30) Chung, S. H.; Hoffmann, A.; Bader, S. D.; Liu, C.; Kay, B.; Makowski, L.; Chen, L. Biological Sensors Based on Brownian Relaxation of Magnetic Nanoparticles. Appl. Phys. Lett. 2004, 85, 2971-2973.

(31) Tu, L.; Jing, Y.; Li, Y.; Wang, J.-P. Real-Time Measurement of Brownian Relaxation of Magnetic Nanoparticles by a MixingFrequency Method. Appl. Phys. Lett. 2011, 98, 213702.

(32) Wu, K.; Tu, L.; Su, D.; Wang, J.-P. Magnetic Dynamics of Ferrofluids: Mathematical Models and Experimental Investigations. J. Phys. Appl. Phys. 2017, 50, 085005. 
(33) Reeves, D. B.; Weaver, J. B. Simulations of Magnetic Nanoparticle Brownian Motion. J. Appl. Phys. 2012, 112, 124311.

(34) Taukulis, R.; Cēbers, A. Coupled Stochastic Dynamics of Magnetic Moment and Anisotropy Axis of a Magnetic Nanoparticle. Phys. Rev. E: Stat., Nonlinear, Soft Matter Phys. 2012, 86, 061405.

(35) Ota, S.; Matsugi, Y.; Nakamura, T.; Takeda, R.; Takemura, Y.; Kato, I.; Nohara, S.; Sasayama, T.; Yoshida, T.; Enpuku, K. Effects of Size and Anisotropy of Magnetic Nanoparticles Associated with Dynamics of Easy Axis for Magnetic Particle Imaging. J. Magn. Magn. Mater. 2019, 474, 311-318.

(36) Nguyen, L.; Phong, P.; Nam, P.; Manh, D.; Thanh, N.; Tung, L.; Phuc, N. The Role of Anisotropy in Distinguishing Domination of Néel or Brownian Relaxation Contribution to Magnetic Inductive Heating: Orientations for Biomedical Applications. Materials 2021, $14,1875$.

(37) Zhao, Z.; Garraud, N.; Arnold, D. P.; Rinaldi, C. Effects of Particle Diameter and Magnetocrystalline Anisotropy on Magnetic Relaxation and Magnetic Particle Imaging Performance of Magnetic Nanoparticles. Phys. Med. Biol. 2020, 65, 025014.

(38) Zhang, X.; Reeves, D. B.; Perreard, I. M.; Kett, W. C.; Griswold, K. E.; Gimi, B.; Weaver, J. B. Molecular Sensing with Magnetic Nanoparticles Using Magnetic Spectroscopy of Nanoparticle Brownian Motion. Biosens. Bioelectron. 2013, 50, 441-446.

(39) Wu, K.; Liu, J.; Saha, R.; Su, D.; Krishna, V. D.; Cheeran, M. C.-J.; Wang, J.-P. Magnetic Particle Spectroscopy for Detection of Influenza A Virus Subtype H1N1. ACS Appl. Mater. Interfaces 2020, 12, 13686-13697.

(40) Orlov, A. V.; Pushkarev, A. V.; Znoyko, S. L.; Novichikhin, D. O.; Bragina, V. A.; Gorshkov, B. G.; Nikitin, P. I. Multiplex Label-Free Biosensor for Detection of Autoantibodies in Human Serum: Tool for New Kinetics-Based Diagnostics of Autoimmune Diseases. Biosens. Bioelectron. 2020, 159, 112187.

(41) Khurshid, H.; Shi, Y.; Berwin, B. L.; Weaver, J. B. Evaluating Blood Clot Progression Using Magnetic Particle Spectroscopy. Med. Phys. 2018, 45, 3258-3263.

(42) Khurshid, H.; Friedman, B.; Berwin, B.; Shi, Y.; Ness, D. B.; Weaver, J. B. Blood Clot Detection Using Magnetic Nanoparticles. AIP Adv. 2017, 7, 056723.

(43) Nikitin, M. P.; Orlov, A. V.; Znoyko, S. L.; Bragina, V. A.; Gorshkov, B. G.; Ksenevich, T. I.; Cherkasov, V. R.; Nikitin, P. I. Multiplex Biosensing with Highly Sensitive Magnetic Nanoparticle Quantification Method. J. Magn. Magn. Mater. 2018, 459, 260-264.

(44) Orlov, A. V.; Burenin, A. G.; Massarskaya, N. G.; Betin, A. V.; Nikitin, M. P.; Nikitin, P. I. Highly Reproducible and Sensitive Detection of Mycotoxins by Label-Free Biosensors. Sens. Actuators, B 2017, 246, 1080-1084.

(45) Znoyko, S. L.; Orlov, A. V.; Pushkarev, A. V.; Mochalova, E. N.; Guteneva, N. V.; Lunin, A. V.; Nikitin, M. P.; Nikitin, P. I. Ultrasensitive Quantitative Detection of Small Molecules with Rapid Lateral-Flow Assay Based on High-Affinity Bifunctional Ligand and Magnetic Nanolabels. Anal. Chim. Acta 2018, 1034, 161-167.

(46) Pietschmann, J.; Voepel, N.; Spiegel, H.; Krause, H.-J.; Schroeper, F. Brief Communication: Magnetic Immuno-Detection of SARS-CoV-2 Specific Antibodies. 2020, bioRxiv 2020.06.02.131102.

(47) Znoyko, S. L.; Orlov, A. V.; Bragina, V. A.; Nikitin, M. P.; Nikitin, P. I. Nanomagnetic Lateral Flow Assay for High-Precision Quantification of Diagnostically Relevant Concentrations of Serum TSH. Talanta 2020, 216, 120961.

(48) Chugh, V. K.; Wu, K.; Krishna, V. D.; di Girolamo, A.; Bloom, R. P.; Wang, Y. A.; Saha, R.; Liang, S.; Cheeran, M. C.; Wang, J.-P. Magnetic Particle Spectroscopy (MPS) with One-Stage Lock-in Implementation for Magnetic Bioassays with Improved Sensitivities. 2021, arXiv:2105.12718. ArXiv Prepr.

(49) Johnson, J. L.; Ordal, E. J. Deoxyribonucleic Acid Homology in Bacterial Taxonomy: Effect of Incubation Temperature on Reaction Specificity. J. Bacteriol. 1968, 95, 893-900.

(50) Schwesinger, F.; Ros, R.; Strunz, T.; Anselmetti, D.; Güntherodt, H.-J.; Honegger, A.; Jermutus, L.; Tiefenauer, L.;
Plückthun, A. Unbinding Forces of Single Antibody-Antigen Complexes Correlate with Their Thermal Dissociation Rates. Proc. Natl. Acad. Sci. 2000, 97, 9972-9977.

(51) Green, D. J.; Litt, G. J.; Laugharn, J. A., Jr Use of High Pressure to Accelerate Antibody: Antigen Binding Kinetics Demonstrated in an HIV-1 P24: Anti-HIV-1 P24 Assay. Clin. Chem. 1998, 44, 341-342.

(52) Penn, M. A.; Drake, D. M.; Driskell, J. D. Accelerated SurfaceEnhanced Raman Spectroscopy (SERS)-Based Immunoassay on a Gold-Plated Membrane. Anal. Chem. 2013, 85, 8609-8617.

(53) Guo, H.; Zhou, X.; Zhang, Y.; Gu, C.; Song, B.; Shi, H. Kinetic Analysis of a High-Affinity Antibody/Antigen Interaction Performed by Planar Waveguide Fluorescence Immunosensor. RSC Adv. 2016, 6, 13837-13845.

(54) Chandrapalan, S.; Persaud, K.; Arasaradnam, R. P. Breath Diagnostics in the Era of SARS-CoV-2-Clinical and Research Arena. J. Breath Res. 2020, 14, 042002.

(55) Ryan, D. J.; Toomey, S.; Madden, S. F.; Casey, M.; Breathnach, O. S.; Morris, P. G.; Grogan, L.; Branagan, P.; Costello, R. W.; De Barra, E.; Hurley, K.; Gunaratnam, C.; McElvaney, N. G.; OBrien, M. E.; Sulaiman, I.; Morgan, R. K.; Hennessy, B. T. Use of Exhaled Breath Condensate (EBC) in the Diagnosis of SARS-COV-2 (COVID-19). Thorax 2021, 76, 86-88.

(56) Li, X.; Li, J.; Ge, Q.; Du, Y.; Li, G.; Li, W.; Zhang, T.; Tan, L.; Zhang, R.; Yuan, X.; Zhang, H.; Zhang, C.; Liu, W.; Ding, W.; Sun, L.; Chen, K.; Wang, Z.; Shen, N.; Lu, J. Detecting SARS-CoV-2 in the Breath of COVID-19 Patients. Front. Med. 2021, 8, 604392.

(57) Quirouette, C.; Younis, N. P.; Reddy, M. B.; Beauchemin, C. A. A. A Mathematical Model Describing the Localization and Spread of Influenza A Virus Infection within the Human Respiratory Tract. PLoS Comput. Biol. 2020, 16, No. e1007705.

(58) Tan, V. Y. J.; Zhang, E. Z. Y.; Daniel, D.; Sadovoy, A.; Teo, N. W. Y.; Kiong, K. L.; Toh, S. T.; Yuen, H. W. Respiratory Droplet Generation and Dispersal during Nasoendoscopy and Upper Respiratory Swab Testing. Head Neck 2020, 42, 2779-2781.

(59) Choi, J.; Jeun, M.; Yuk, S.-S.; Park, S.; Choi, J.; Lee, D.; Shin, H.; Kim, H.; Cho, I.-J.; Kim, S. K.; Lee, S.; Song, C. S.; Lee, K. H. Fully Packaged Portable Thin Film Biosensor for the Direct Detection of Highly Pathogenic Viruses from On-Site Samples. ACS Nano 2018, $13,812-820$.

(60) Wu, F.; Yuan, H.; Zhou, C.; Mao, M.; Liu, Q.; Shen, H.; Cen, Y.; Qin, Z.; Ma, L.; Song Li, L. Multiplexed Detection of Influenza A Virus Subtype H5 and H9 via Quantum Dot-Based Immunoassay. Biosens. Bioelectron. 2016, 77, 464-470.

(61) Bai, Z.; Wei, H.; Yang, X.; Zhu, Y.; Peng, Y.; Yang, J.; Wang, C.; Rong, Z.; Wang, S. Rapid Enrichment and Ultrasensitive Detection of Influenza A Virus in Human Specimen Using Magnetic Quantum Dot Nanobeads Based Test Strips. Sens. Actuators, B 2020, 325, 128780.

(62) Zhang, Y.; Wang, X.-P.; Perner, S.; Bankfalvi, A.; Schlücker, S. Effect of Antigen Retrieval Methods on Nonspecific Binding of Antibody-Metal Nanoparticle Conjugates on Formalin-Fixed ParaffinEmbedded Tissue. Anal. Chem. 2018, 90, 760-768.

(63) Nance, E. A.; Woodworth, G. F.; Sailor, K. A.; Shih, T. Y.; Xu, Q.; Swaminathan, G.; Xiang, D.; Eberhart, C.; Hanes, J. A dense poly(ethylene glycol) coating improves penetration of large polymeric nanoparticles within brain tissue. Sci. Transl. Med. 2012, 4, 149ra119.

(64) Schneider, C. S.; Perez, J. G.; Cheng, E.; Zhang, C.; Mastorakos, P.; Hanes, J.; Winkles, J. A.; Woodworth, G. F.; Kim, A. J. Minimizing the Non-Specific Binding of Nanoparticles to the Brain Enables Active Targeting of Fn14-Positive Glioblastoma Cells. Biomaterials 2015, 42, 42-51. 